\title{
Robust Prior Stage Epileptic Seizure Diagnosis System using Resnet and Backpropagation Techniques
}

\author{
Priti N. Bhagat ${ }^{1}$, K.S.Ramesh ${ }^{2}$,Dr.Venu Gopala Rao Matcha ${ }^{3}$, S T Patil ${ }^{4}$ \\ 1Research Scholar, KoneruLakshmaiah Education Foundation, Vaddeswaram, Guntur, Andhra Pradesh, India, \\ bhagatpnh@gmail.com \\ 2 Professor, KoneruLakshmaiah Education Foundation, Vaddeswaram, Guntur, Andhra Pradesh, India, \\ dr.ramesh@kluniversity.in \\ 3 Professor, KoneruLakshmaiah Education Foundation, Vaddeswaram, Guntur, Andhra Pradesh, India, \\ mvgr03@kluniversity.in \\ 4 Professor Vishwakarma Institute of Technology, Pune, Maharashtra, India, stpatil77@ gmail
}

\begin{abstract}
The human Brain generates the Electroencephalogram (EEG) signals, which contains physiological information. This EEG oriented the human brain activities, making it utilized for epileptic seizure identification and diagnosis. The present epilepsy visual techniques take a large amount of time for inspection of EEG data. The human proficient is identified epilepsy, but slow diagnosis happens. Therefore patient may often inconsistence stage; it sometimes causes deaths. In this investigation, an advanced early-stage epileptic seizure identification and classification framework has developed. Which includes two steps, at first step EEG signal has preprocessed with ResNet deep learning mechanism. This technique calculated the abnormal signal identification at alpha, beta, gamma and delta waveforms. These observations find out the patient early-stage epileptic seizure detection. The ResNet learning model trains the suspicious raises in the EEG signal, but clear classification required. Thus moving to the second stage, in this backpropagation (BP) auto stack encoder is classifying epilepsy efficiently. The dataset is a real-time clinical EEG database collected from practical and meaningful epilepsy patients. For testing and training, CHB-MIT datasets are selected for the proposed framework, and proposed ResNet and BP achieved a classification accuracy of $99.83 \%$ and throughput $99.72 \%$.
\end{abstract}

Key words : EEG, ResNet, BP auto stack, Epileptic seizure, accuracy.

\section{INTRODUCTION}

The EEG signal can help the detection of epilepsy using a brain-computer interface and communication system based on brain activities. The EEG signal is a key factor to identify the features of abnormal conditions. The epilepsy EEG signal components consist of noise, using various segmentation and filtering methods remove the original information. The principal component analysis (PCA) is a dimension reduction process for extracting the signals without losing the data. PCA can enhance the artefacts in the extracted signal and provides better performance when we move to decompose the EEG signal [1]. The epileptic seizure supportive information evidence the diagnosis process and to help the seizure classification. The patterns in the EEG has detected the neuroimages and laboratory images based on machines such data can quickly identify the epilepsy detection efficiently. The real-time clinical data trains can give the diagnosis phase with less accurate.

The previous classifications automatically identify epilepsy, but the prior stage of detection is not available [2]. The human brain mapping is related to EEG data; it reflects the connectivity of Alzheimer's and seizure patients. When the level of EEG interval synchronizes with an experimental dataset, then automatically seizure spikes signals are identified. This shootout successfully determines the prior stage of epileptic seizure [3]. Functional connectivity and signal space normalization on gamma waveform give the patient information experimentally. Along with gamma signal theta, alpha and lambda waveform analysis provides the full scale high dimensional seizures efficiently [4]. The phase transfer entropy with oscillation measures the direct connectivity of EEG seizures; the noise samples have extracted by time-series filtering across un-validated EEG seizures. The transfer entropy is a well suited estimated model for epileptic seizure prior stage of diagnosis [5].

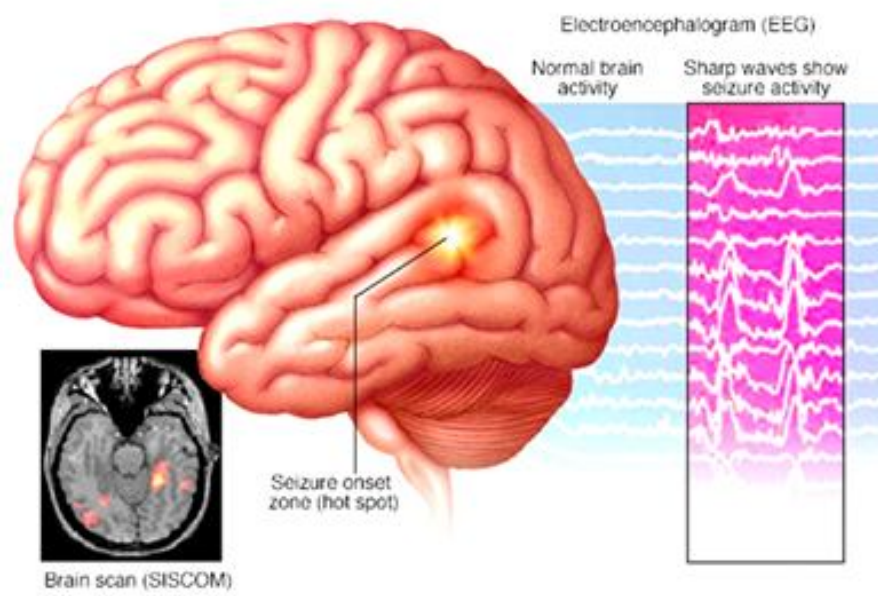

Figure 1: Example model of EEG generation. 
Priti N. Bhagat et al., International Journal of Emerging Trends in Engineering Research, 8(5), May 2020, 2214 - 2222

Figure 1 demonstrates that the general model of EEG samples collected from human interaction; this work comes under neuroscience and signal extraction studies. Around the world, the EEG classification framework allows for easy access and diagnosis of epileptic patients. The machine learning and feature extraction give the fundamental elements of epileptics diagnosis. The feature extraction, selection, data processing and classification is a process to estimate the seizures of patients. The horizontal visibility graph model estimates the time series between robust EEG intervals. The periodicity of the input signal has been preprocessed with differential wavelet transforms and apply the phase-based transfer entropy at $8.0-13.0 \mathrm{~Hz}$ alpha band. This study gives the direct classification of extensive brain network and its epileptic information. The anterior and posterior information with phase entropy compared with experimental pattern dataset, thus generate the abnormal EEG signal conditions [7]. The study of non-epileptic seizures and psychogenic decomposition signal extraction process, pre-estimate the EEG signal features. In the next phase, SVM-RBF linear models classify the epileptic seizures with reported results. This highly organized metrics maximize the classification performance; the frequency spectrum can quickly identify the epileptic seizures by applying the SVM-RBF technique [8].

The main objective of epileptic seizure prediction is a neurological disorder identification of epileptic patients. This work analyzes the ictal rules on $460 \mathrm{hrs}$ patients data, for $42 \mathrm{hrs}$ application of SVM get sensitivity is $91.83 \%$ to $96.63 \%$. With this false prediction, the rate is around $0.08 \mathrm{hrs}$ has been identified. The presented ictal-SVM algorithm attains better performance and robust [9].

\subsection{Biomedical EEG information:}

EEG data can generate the electrical activity of the Brain. The recorded signals reflect the operation of the human Brain and the electrical property of humans. The signal intensity of the EEG signal is in microvolts; this waveform contains many frequencies, i.e. Delta, Theta, Alpha and Beta.

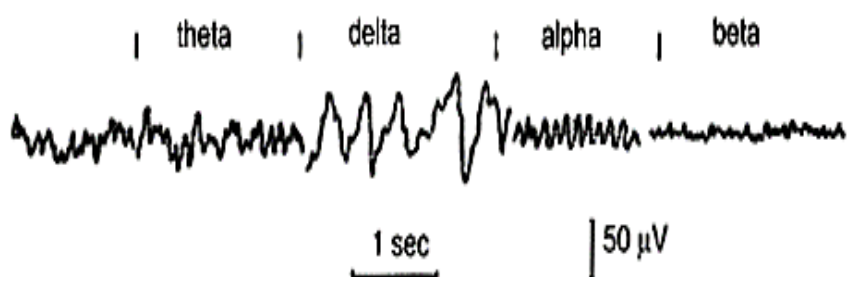

Figure 2: Estimation of EEG signal.

The variable frequencies are presented in the above figure 2 , it refers to the activity of 1) Rhythmic 2) Arrhythmic and 3) Dysrhythmic components. The first one approximately constant in frequency, the second one is no stability of frequency is presented, and the third is related to patient groups are healthy subjects.

\section{Delta:}

It is a frequency of $\leq 3 \mathrm{~Hz}$; it is the highest amplitude signal focuses on the metabolic conditions of patients.
Theta:

This signal has 3.5 to $7.5 \mathrm{~Hz}$ 's, and it classifies the patients "slow" activity, up to 13 years this signal is normal in nature. But, abnormal in awake of adults.

\section{Alpha:}

It has a frequency of 7.5 to $13 \mathrm{~Hz}$; it appears when a patient closes the eyes and relaxation condition. When the patients open their eyes or alerting by anyone, then automatically this alpha signal disappears.

\section{Beta:}

It is a "fast" activity $>14 \mathrm{~Hz}$, it usually reduced or absent when the patient was going to cortical damage. Also, it is an indication of anxious, alert and their eyes open.

The above all discussion related to bio-medical signal acquisition and its applications for researchers [10]. The main aim of this work is the computational solutions for EEG data and an indication of 10minutes prior to the detection of seizures in patients. Hence it is necessary to implement a computer-aided design for the diagnosis of automatic abnormal activity detection of seizure patients. The non-linear abnormal patients and their activities are estimated by CAD design. The prediction of epileptic seizures diagnosis is an unstable model, and these are impacts of an uncontrolled, careless and unhealthy diet of patients. The Lyapunov exponent algorithms are capable of finding the fractional-order epileptic signals and their characteristics. This experiment consists of noisy signals; it creates the discontinuities in the reliable prediction of seizures [11].

\begin{tabular}{|l|l|l|}
\hline $\begin{array}{l}\text { Refe- } \\
\text { rence } \\
\text { No }\end{array}$ & Technique & Key points \\
\hline$[12]$ & $\begin{array}{l}\text { Probability } \\
\text { density } \\
\text { function }\end{array}$ & $\begin{array}{l}\text { Based on gain decision } \\
\text { identifies the falls alarm in the } \\
\text { EEG signal. } \\
\text { The sequence of filtering process } \\
\text { predicts the seizures of the } \\
\text { patient. }\end{array}$ \\
\hline$[13]$ & $\begin{array}{l}\text { The common } \\
\text { spatial pattern } \\
\text { on CHB-MIT }\end{array}$ & $\begin{array}{l}\text { This experiment consists of a } \\
\text { seizure prediction of 24 patients } \\
\text { record from CHB-MIT database. } \\
\text { With this attain 89\% sensitivity, } \\
\text { 39\% false prediction in the time } \\
\text { of 69 minutes/ 120 minutes. }\end{array}$ \\
\hline$[14]$ & $\begin{array}{l}\text { Phase locking } \\
\text { value on 65 } \\
\text { clinical cases } \\
\text { are analyzed } \\
\text { with the } \\
\text { imperial mode } \\
\text { decomposition } \\
\text { method. }\end{array}$ & $\begin{array}{l}\text { The phase-locking value } \\
\text { technique achieves 99.8\% noise } \\
\text { exhausted signal. } \\
\text { The classification rate achieved } \\
\text { as 83.27\% on alpha, beta, } \\
\text { gamma and theta signals. }\end{array}$ \\
\hline
\end{tabular}


Priti N. Bhagat et al., International Journal of Emerging Trends in Engineering Research, 8(5), May 2020, 2214 - 2222

\begin{tabular}{|c|c|c|}
\hline [15] & $\begin{array}{l}\text { Phase/Amplitu } \\
\text { de Lock values } \\
\text { Mechanism }\end{array}$ & $\begin{array}{l}\text { The CHB-MIT EEG datasets } \\
\text { extract the seizures for testing } \\
\text { the diagnosis patient. } \\
\text { The evaluation of this algorithm } \\
\text { calculates the performance } \\
\text { measures such as sensitivity and } \\
\text { precision. }\end{array}$ \\
\hline [16] & $\begin{array}{l}\text { Sensor } \\
\text { Medium } \\
\text { access control } \\
\text { based } \\
\text { monitoring } \\
\text { epilepsy } \\
\text { patients }\end{array}$ & $\begin{array}{l}\text { The epileptic seizer's } \\
\text { patients(EP) are facing many } \\
\text { problems at diagnosis process. } \\
\text { So, this work proposes a WSN } \\
\text { based EP application, and it has } \\
\text { designed on the NS2 Simulation } \\
\text { Tool. }\end{array}$ \\
\hline [17] & $\begin{array}{l}\text { The MEG and } \\
\text { EEG signals } \\
\text { have analyzed } \\
\text { by Regional } \\
\text { difference } \\
\text { Ratios } \\
\text { technique }\end{array}$ & $\begin{array}{l}\text { Depending upon the signal to } \\
\text { noise ratio technique } \\
\text { differentiate the seizers in the } \\
\text { dataset. } \\
\text { The frontal area and spike } \\
\text { sensitivity can easily predict the } \\
\text { epileptic seizers in the patient } \\
\text { data. }\end{array}$ \\
\hline [18] & $\begin{array}{l}\text { Multidimensio } \\
\text { nal } \\
\text { normalization } \\
\text { and machine } \\
\text { learning } \\
\text { technique on } \\
\text { EEG data }\end{array}$ & $\begin{array}{l}\text { In } 740 \text { hours of EEG data, } \\
\text { predicts the seizure of patients. } \\
\text { The prediction accuracy is } \\
\text { around } 74 \% \text { had attained. }\end{array}$ \\
\hline [19] & $\begin{array}{l}\text { Relative } \\
\text { power spectral } \\
\text { features }\end{array}$ & $\begin{array}{l}\text { The relative combination of } \\
\text { subband multichannel EEG } \\
\text { signals is analyzed with a } \\
\text { relative spectral algorithm. } \\
\text { The sensitivity of around } 76 \% \\
\text { percentage and false prediction } \\
\text { rate by } 0.1 \text { per hour. } \\
\text { It can possible by the application } \\
\text { of machine learning techniques } \\
\text { on reduced feature extracted } \\
\text { seizures data. }\end{array}$ \\
\hline [20] & $\begin{array}{l}\text { Common } \\
\text { spatial pattern } \\
\text { technique }\end{array}$ & $\begin{array}{l}\text { Mutual information-based } \\
\text { individual feature extraction on } \\
\text { EEG data. } \\
\text { With this algorithm attains } \\
56.9 \% \text { means for selecting } \\
\text { MIB-IF dataset. }\end{array}$ \\
\hline
\end{tabular}

Epileptic seizure patients always suffer from prior diagnosis and treatment. Continuous medication or surgical procedures can handle epileptic patients. More than 39\% of the patients died because of unusual medications or treatment; these do not control the seizure activity [21]. The computational methods for epileptic seizures using machine learning techniques solve the major issues, i.e. time of anticipation and True positive rate. With this method, the True positive rate is 0.922 and time of anticipation is 32 minutes has achieved. This investigation selects the 22 patients data at CHB-MIT database. The CHB-MIT database consists of this type of samples, which are shown in above Figure. Using these samples, apply the machine learning mechanisms, to identifies the seizures in the EEG signals [22]. The detection of EEG signal disorders can help the patients at fast diagnosis. The deep learning techniques with convolutional neural networks are extracted the problem identification efficiently [23].
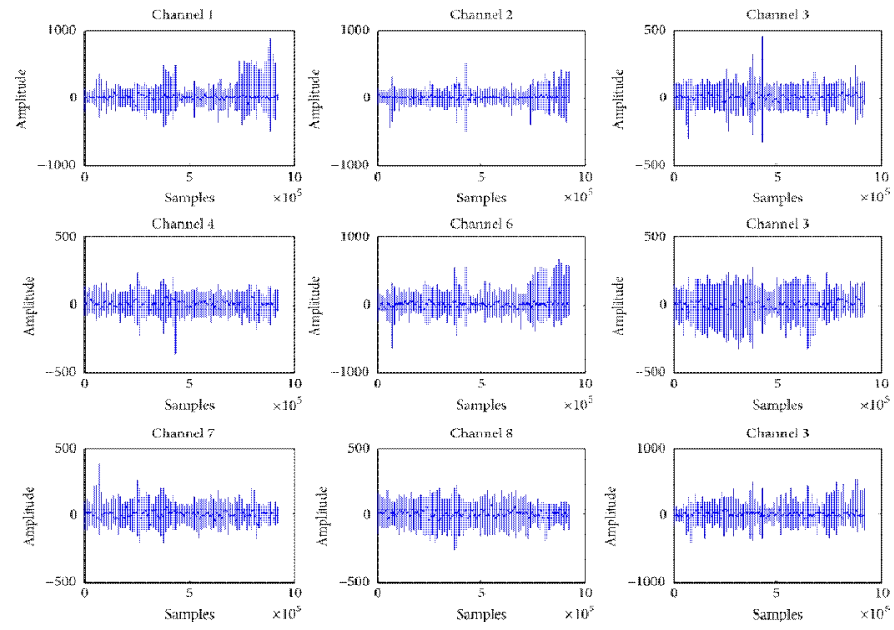

Figure 3: sample EEG signals.

The above all literature survey shootout the problems of epileptic seizure patient diagnosis and their issues at the treatment stage. The continuous research on medical diagnosis can give better achievement compared to existed methods. Therefore we further investigate the prior stage of epileptic seizure diagnosis using deep learning mechanisms.

\section{METHODOLOGY}

The proposed methodology mainly classified into two phases;in the first phase, the backpropagation (BP) auto stack encoder (ASE) is working as pre-processor. Coming to second phase ResNet deep learning technique is used as a classifier. The combination of preprocessing and classification gives a better diagnosis for epileptic patients at a prior stage.

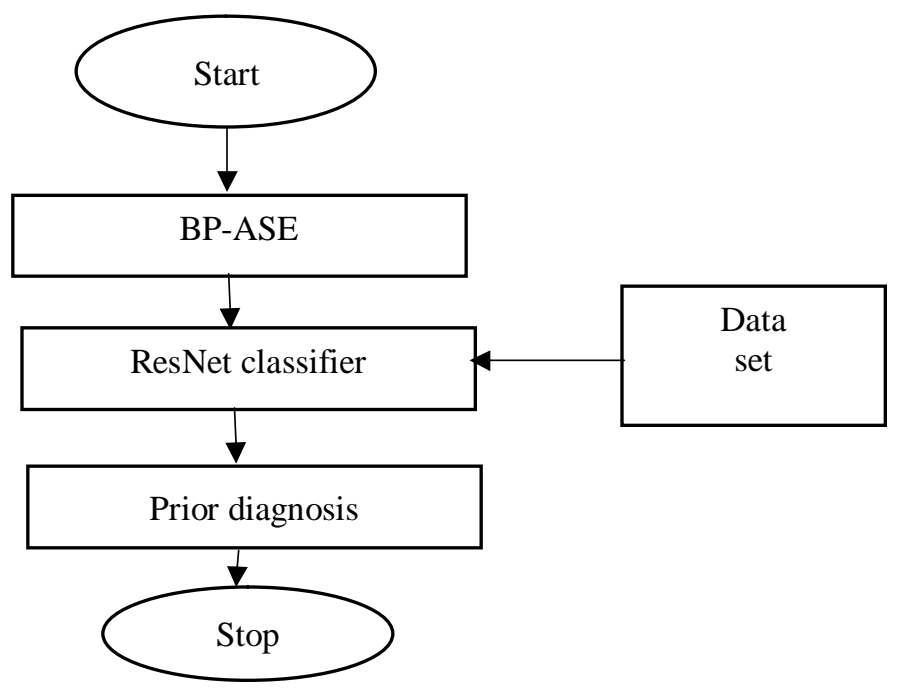

Figure 4: Proposed BP-ASE-ResNet block diagram. 
As shown in figure 4, the block diagram of the proposed BP-ASE ResNet design for automatic diagnosis of epileptic patients. It is an unsupervised learning process and solves the critical seizure issues presented in the EEG signal. The ability of testing, training and classification process accurate the designed application.

\section{Dataset:}

In this investigation, various orientations of different age group EEG datasets are collected from CHB-MIT database.

Available link: https://physionet.org/content/chbmit/1.0.0/ This database collected the children, adults and old age people EEG signal recordings. In this records 23 major cases are collected, i.e. 5-males (age 3-22) and 17-females (age-2-19). Each case consists of the file name as CHB01, CHB02 and....CHB0N, and also each internal file consists of 9-42 continuous .edf files. These datasets are trained with real-time clinical available data.

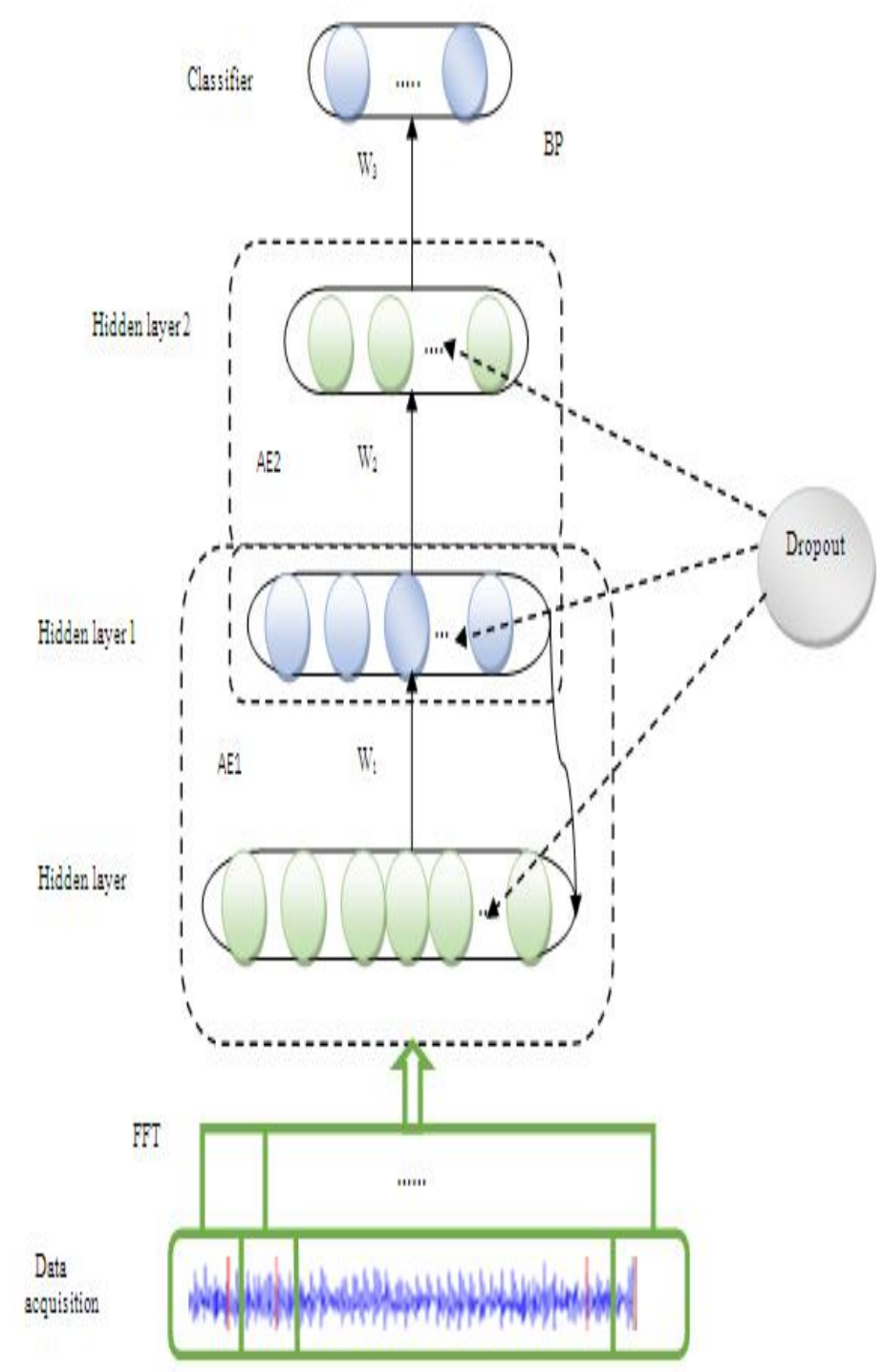

BP-ASE:

The Back propagation-based auto stack encoder system consists of " $\mathrm{i}$ " hidden layers can tune the structure of pre-processor.

The input data samples are applied to autoencoder and getting outputs as learned vectors. This vectors applied to input as layered backpropagation network and extracted the features until the training process complete. After the training process, all hidden layer information has attained by the backpropagation algorithm. This process reduced the fitness function and updated the parameters to achieve excellent training. The figure 5 describes that BP-ASE functional diagram, which is working as pre-processor and features extractor. At the first step, apply the EEG signal samples for prediction of seizures. At this stage, FFT can handle the data acquisition from available signals. After the parameter attainment, we are applying the backpropagation mechanism, based on a weight balancing approach.

The signal training set collected from the following composing set $\left\{X_{n}\right\}_{n=1}^{N}$.

$$
\begin{gathered}
h_{n}=f\left(W_{1} x_{n}+b_{1}\right)---(1) \\
\hat{x}_{n}=g\left(W_{2} h_{n}+b_{2}\right)--(2) \\
\emptyset(\theta)=\underset{\theta, \theta^{1}}{\operatorname{argmin}} \frac{1}{n} \sum_{i=1}^{n} L\left(x^{i}, \hat{x}^{i}\right)--(3)
\end{gathered}
$$

Where $\mathrm{h}_{\mathrm{n}}=$ impulse function of BP auto stack encoder, $\hat{x}_{n}=$ input samples, $\varnothing(\theta)=$ entropy of particular instance. Here $\mathrm{N}=$ number of samples available, $\mathrm{n}=\mathrm{nth}$ sample consist of Fourier coefficient. After the FFT process weights are assigned to the input layer. The un-label training set $\left\{\mathrm{X}^{\mathrm{i}}\right\}_{\mathrm{i}=1}{ }^{\mathrm{M}}$ is applied to hidden layers. When the classifier attains the entropy value, the next process applied to ResNet block, else backpropagation performed until features are extracted. This process differentiates the CHB01, CHB02...CHB0N, after this preprocessing EEG signals, are trained by ResNet learning mechanism.

\section{ResNet Deep Learning:}

The ResNet is nothing but residual network design which can investigate the problem more profoundly. This immediate process understands the theoretical issues and adding additional layers for increasing robustness applications. Progress the performance measures compared to conventional mechanisms.

Figure 5: Proposed BP-ASE. 
Priti N. Bhagat et al., International Journal of Emerging Trends in Engineering Research, 8(5), May 2020, 2214 - 2222

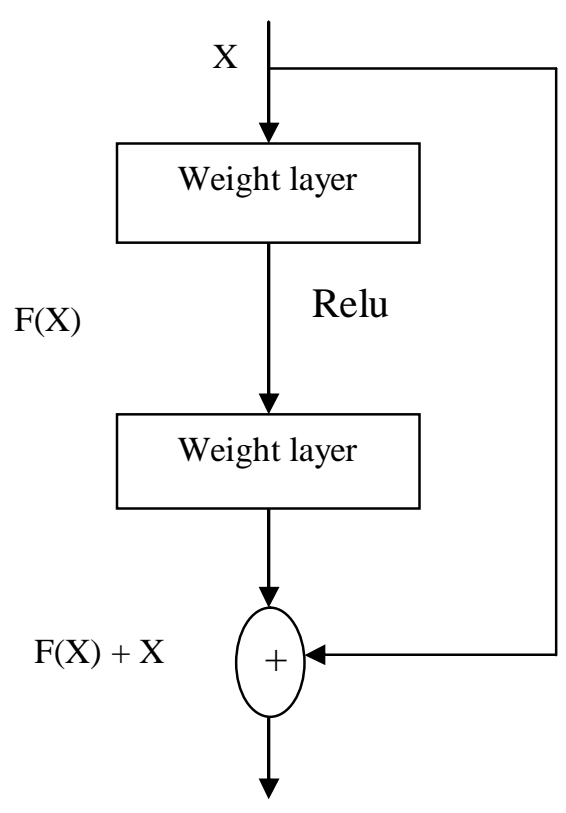

a residual block

Figure 6: Residual block diagram.

As figure 6 shown Residual network functionality, it is utilized to perform the operations depending on weight layers, when the weight layer less than the weight layer+1 then automatically activation function hold the process and pick the sudden change in the data sample. The ResNet internal architecture is classifying the seizure patients by using convolution neural networks. The three blocks of residual networks with convolutional layers $1 \times 1$ gives the entropy values i.e. $\emptyset(\theta)$. If this $\emptyset(\theta)$ value $\leq \mathrm{y}$ then the ResNet network handles the seizures in feedback manner. Else, the activation functions handover the control and identifies the false alarm. When we are using the clinical dataset and collected CHB-MIT dataset, the ResNet and BP-ASE continuously extract the features and classifies the seizure in the EEG signal. When the seizure crosses the entropy value, then ResNet automatically identifies the rise signal and track the interval. This entire phenomenon automatically performed by the proposed BP-ASE and ResNet deep classifiers.

Figure7. Explains about generalized ResNet model, in this model weight layer and activation layers have utilized to generate the fitness activation function. In figure 7 left part to demonstrate the widespread process of ResNet function. Coming to the right part one feedback loop continually sense the model, if $F(x)$ is present in the design means, $1 \mathrm{x} 1$ convolutional feature is coming into the picture along with this ReLu networks deals the issues in the selected EEG samples.

$$
\begin{aligned}
& H_{l}=\operatorname{ReLU}\left(b_{l} * f_{l}\left(H_{l-1}\right)+i d\left(H_{l-1}\right)\right) \\
& H_{l}=\operatorname{ReLU}\left(i d\left(H_{l-1}\right)\right)
\end{aligned}
$$

The equation $4 \& 5$ continuously monitors the EEG signal with fixed intervals, when any sudden change observed in the signal the ReLu function handover the entire operation and identifies the time of instance.

Here $\mathrm{H}_{1}=$ current time of instance,
$H_{l-1}=$ time-shifting signal instance.

$\mathrm{b}_{1}=\mathrm{EEG}$ signal weight.

The $3 \times 3$ convolution can extract the samples in the EEG signal, but when any prior instances of EEG signal extraction required than necessary to add the feedback convolution network. This mathematical computation can extract the EEG signal disorders identifies quickly. The batch norm is the technique which can normalize the EEG samples of real-time clinical data. The Batch norm rescales the available samples and recenters the signals by using stochastic gradient features.

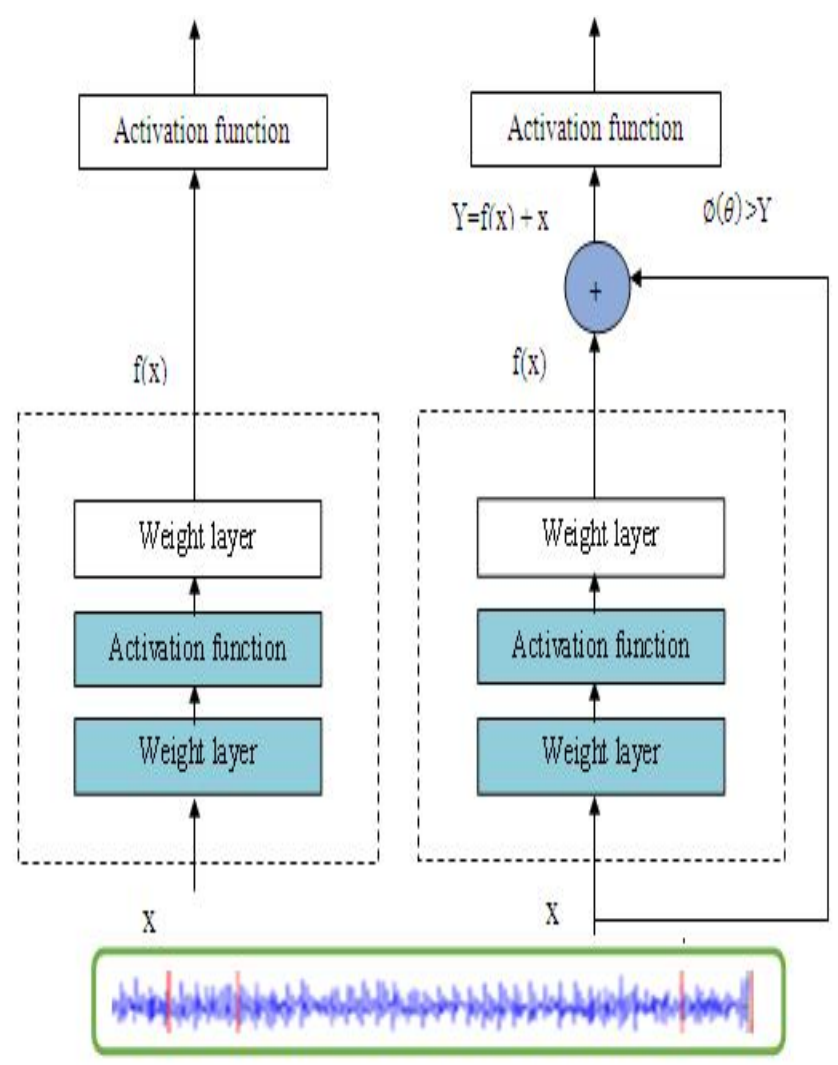

Figure 7: Left: general Resnet Right :Convolution ResNet.

$$
\begin{gathered}
B_{l}=\operatorname{ReLu}\left(p_{l} * f_{l}\left(H_{l-1}\right)+i d\left(H_{l-1}\right)\right)----(6) \\
B_{l}=i d\left(H_{l-1}\right)
\end{gathered}
$$

The equation $6 \& 7$ clearly explains about batch norm function mathematical computations. When batch norm function satisfies the $3 \times 3$ convolutional block, then normalization has performed on EEG signal. The above figure 10 clearly explains about suspicious change in the EEG signal. This suspicious signal further applied to deep ResNet block, which is shown in the above figure 10 . The detected signal is superating from suspicious EEG signal and further, apply the deep ResNet function continuously. Resulting that identifies the lower control limit, upper control limit and detected peak in EEG of seizure patient. In this work, $\mathrm{H}$ is the hidden layer and $\mathrm{X}$ is the pooling layer in the deep ResNet learning model.

$$
\begin{aligned}
H_{l} & =\operatorname{ReLu}\left(p_{l} * f_{l}\left(H_{l-\cdot 1}\right)+i d\left(H_{l-1}\right)\right) \\
p_{l} & =1-\frac{l}{L}\left(1-p_{L}\right)
\end{aligned}
$$


Priti N. Bhagat et al., International Journal of Emerging Trends in Engineering Research, 8(5), May 2020, 2214 - 2222

$$
f_{F}^{*}:=\operatorname{argmin}(X, Y, f) \text { subject to } f \in F \text {------ }(10)
$$

The equation $8,9 \& 10$ clearly explains about ReLu function and its classification model. The ReLu function is the rectified linear network which can handle the EEG signal in piecewise manner. The output has directly extracted from input and impulse response, compared to all deep learning models this ResNet achieves better performance.

$* * * p_{l}=$ probability of seizures in the EEG

$* * f_{F}^{*}=$ argument function concerning classification EEG data. The above all calculations can quickly identify the epileptic seizers in the patients in the prior stage. This gradient function and deep neural networks solve the sudden changes in the EEG data. This entire analysis has smoothly shown in below results section

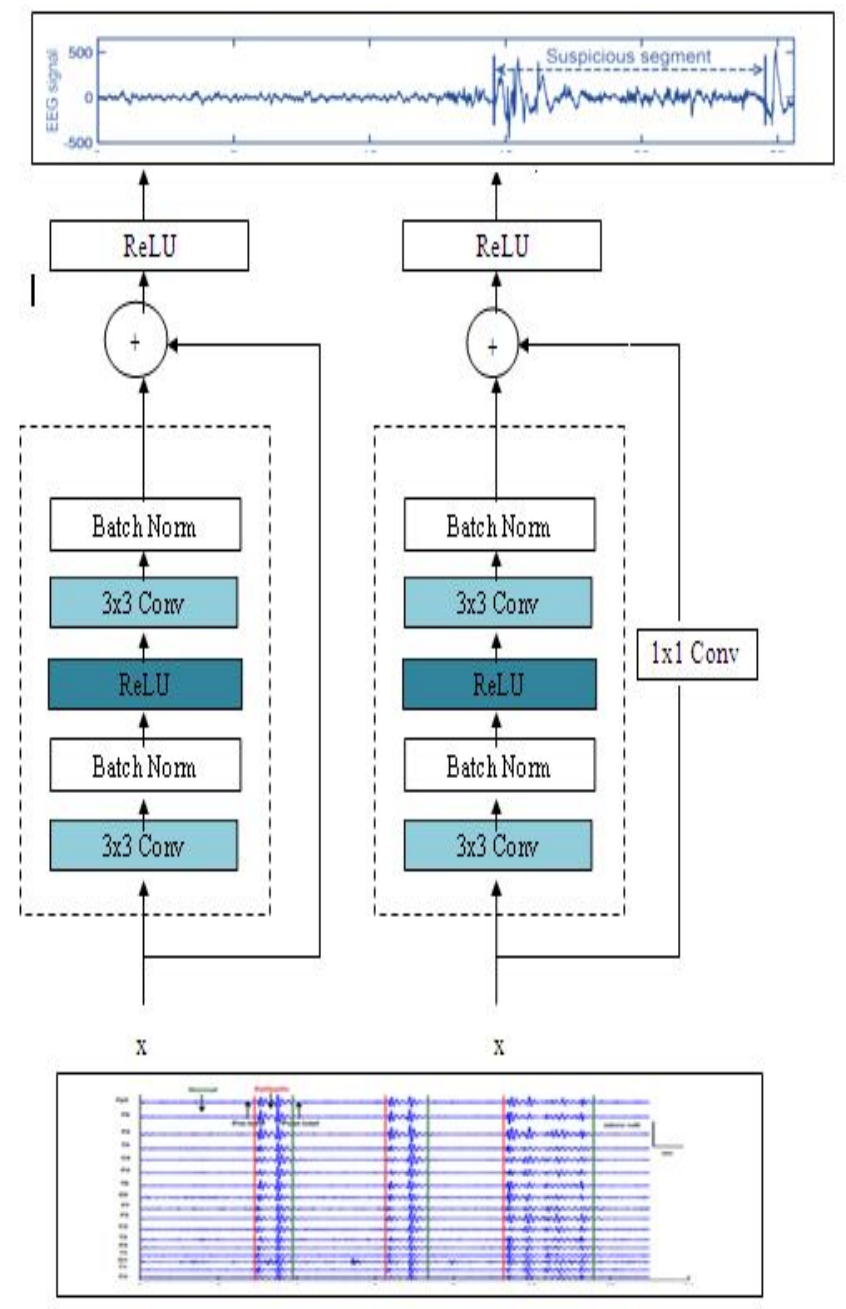

Figure 8: Left: regular ResNet block; Right: ResNet block with $1 \mathrm{x} 1$ convolution

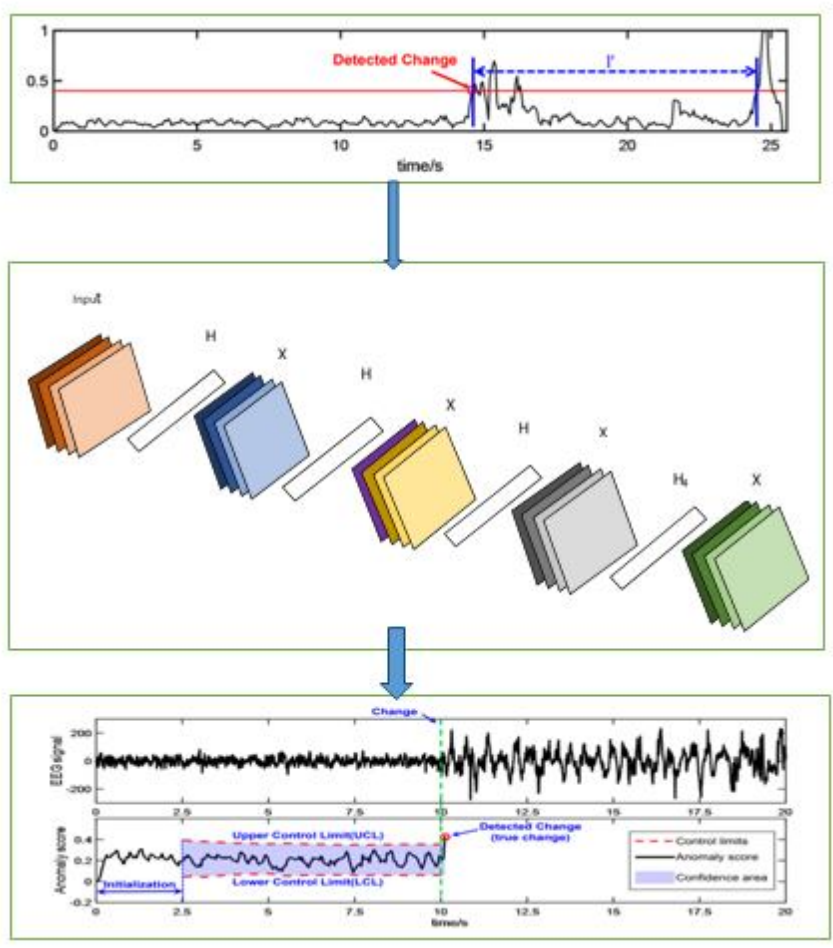

Figure 9: The deep ResNet learning model.
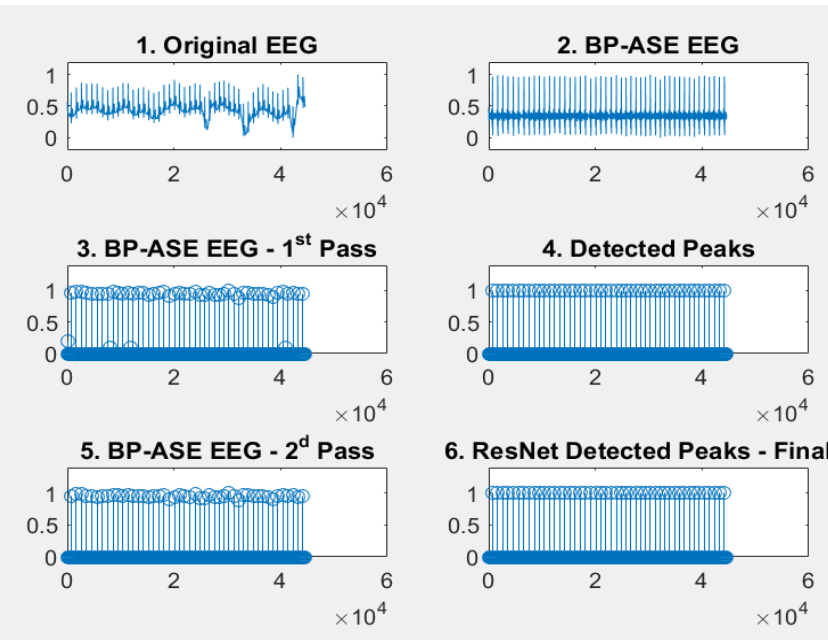

6. ResNet Detected Peaks - Finally

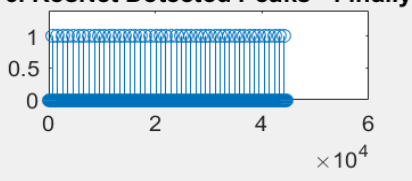

Figure 10: EEG epileptic seizure detection.

The above figure 10 clearly explains about seizures detection process in the selected EEG signal. The entire mathematical steps are divided into six phases, in which the first window is the original EEG sample collected from the clinical database. At the second phase, BP-ASE operation performed on the first window such that peaks are identified easily. In the next step, BP-ASE trained the $1^{\text {st }}$ phase model detects the entire peaks in the EEG signal. Following the third step detected peaks are framed in the fourth window, the second pass estimated the BP-ASE and further estimates the prior EEG signal disorders. At the final step, ResNet classifies the EEG signal and identifies the prior stage of an epileptic seizure. 
Priti N. Bhagat et al., International Journal of Emerging Trends in Engineering Research, 8(5), May 2020, 2214 - 2222

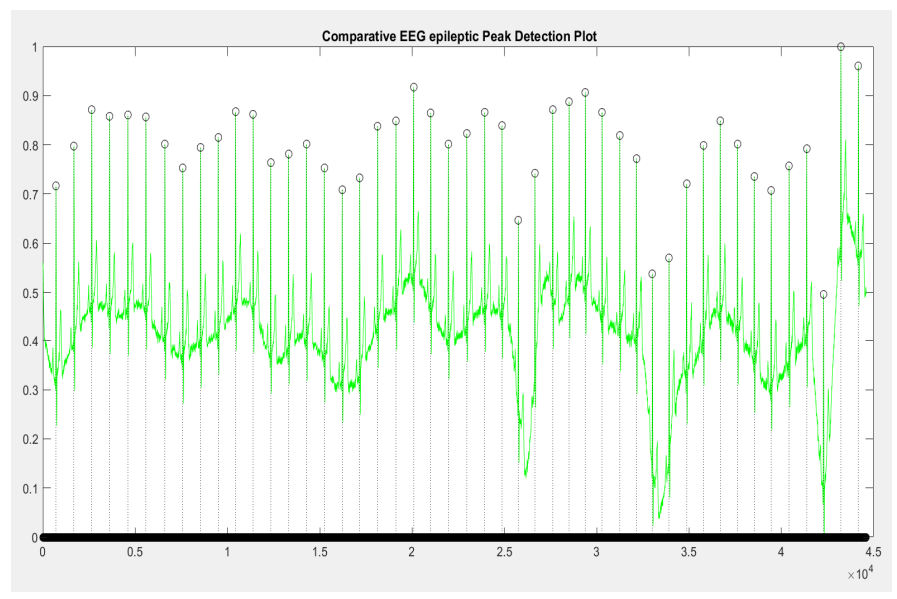

Figure 11: peak detection plot.

As in figure11 falls alarmed, peaks are identified using ResNet deep learning process; this indication alert the patients for epileptic seizures. This is an accurate diagnosis process for a prior stage of seizure identification. The $\mathrm{x}$-axis indicates the time instances of EEG data, and y-axis represents the amplitude of seizure patients.

Figure 12 explains that second sample EEG data analysis, in this ResNet detection and BP-ASE EEG signals easily identify the seizures. This process is continuous monitoring

on EEG patients; hence the prior stage of diagnosis is attained for better treatment.
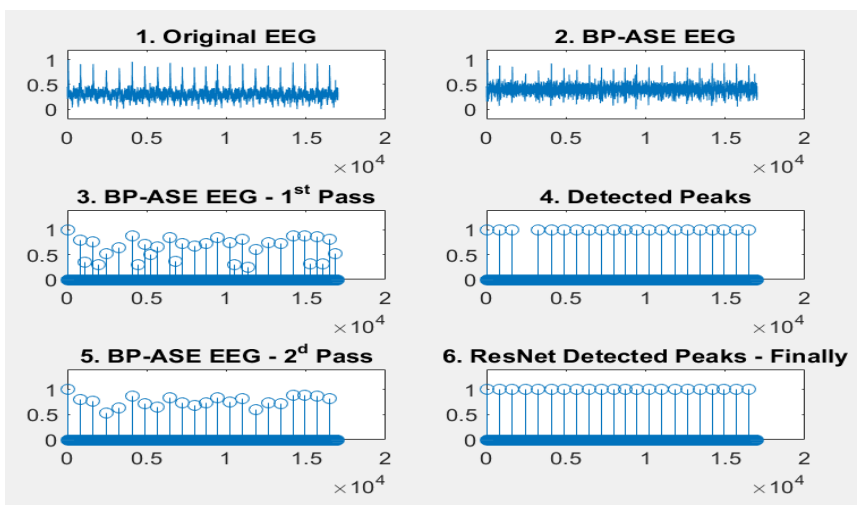

Figure 12: second sample of EEG data.

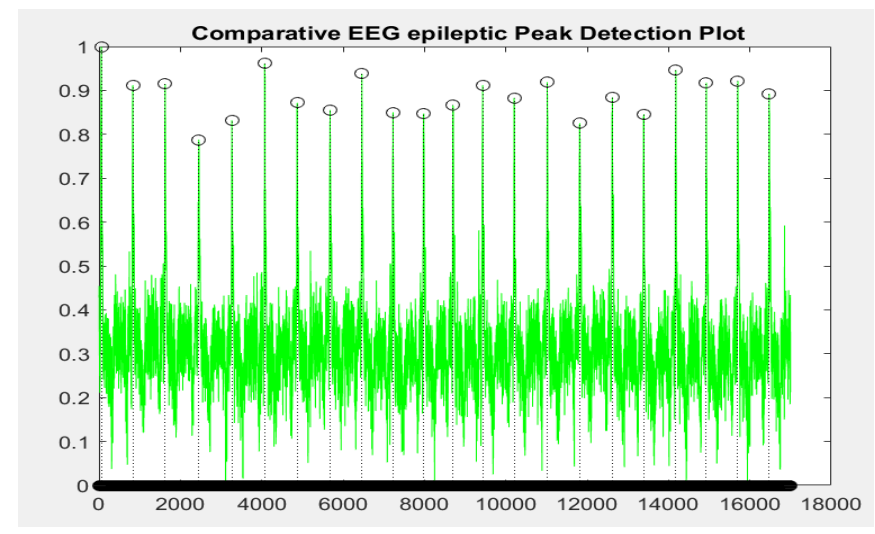

Figure 13: Second sample peak detections.
The figure 13 clearly explains about peak seizures of EEG patients; it is clearly identified that when the entropy of signal crosses the limit then automatically shootout the unnecessary rises in EEG signal. The outcome clearly explains the seizures of EEG dataset.

Table 1: Database

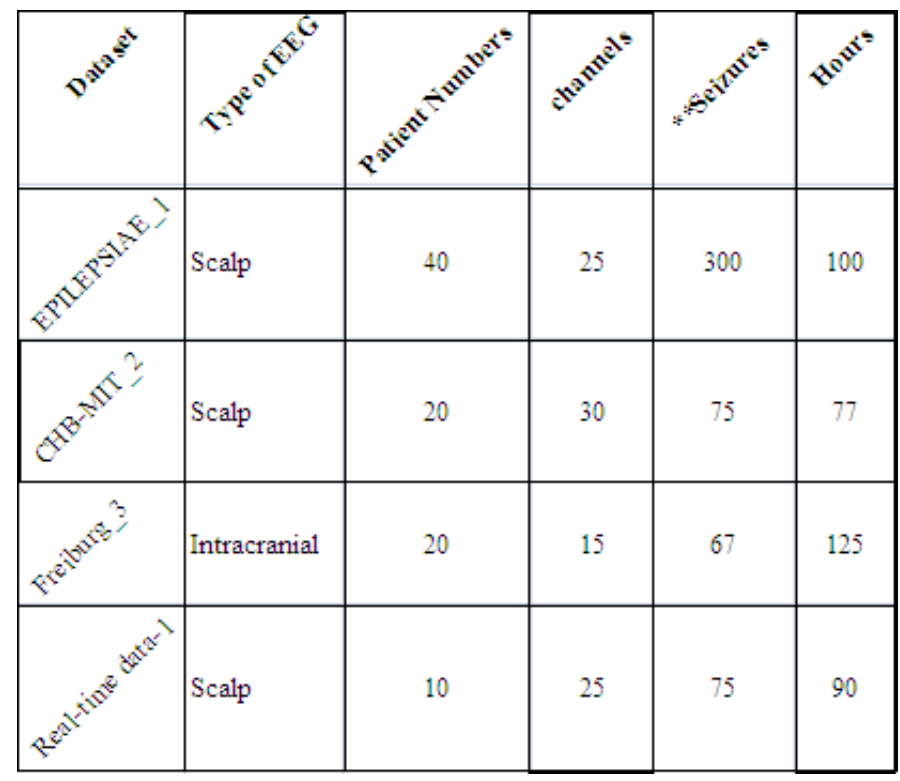

Table 1 demonstrate that the database information for the proposed experiment, the above all database such as epilepsy 1, CHB-MIT, Freiburg and Real-time data are collected. The different patient's various orientations and age groups are preferred for the experiment.

\section{Performance measures:}

1. $R M S E P=\sqrt{\frac{\sum_{i=1}^{n}\left(y_{i}-\hat{y}_{i}\right)^{2}}{n}}$ $\frac{R M S E P\left(i^{*}\right)}{R M S E P(i)} \geq \lambda$

2. NMSE $=\frac{\sum_{i=1}^{n}\left(y_{i}-\hat{y}_{i}\right)^{2}}{y_{i}{ }^{2}}$

3. $\hat{\beta}_{\text {enet }}=\left(1+\frac{\lambda_{2}}{n}\right)\left\{\arg _{\beta} \min \left\|\lambda-\sum_{j=1}^{p} x_{i} \beta_{j}\right\|^{2}+\lambda_{1}\|\beta\|_{1}+\right.$ $\left.\lambda_{1}\|\beta\|_{2}^{2}\right\}$

4. Accuracy $=\hat{\beta}_{\text {enet }}+R_{\text {adj }}^{2} /=\hat{\beta}_{\text {enet }}+R_{\text {adj }}^{2}+\mathrm{F}_{\mathrm{p}}+\mathrm{F}_{\mathrm{n}}$.

5. $\mathrm{F}_{1}$ score $=\mathrm{F}_{\mathrm{p}}+\mathrm{F}_{\mathrm{n}} / / \mathrm{T}_{\mathrm{p}}$.

6. $\mathrm{T}_{\mathrm{p}}=1-\frac{S S R}{S S T}$.

The above all formulas are explains about performance measures of designed applications. The accuracy, f1 score, sensitivity and specificity are shown clearly. 
Priti N. Bhagat et al., International Journal of Emerging Trends in Engineering Research, 8(5), May 2020, 2214 - 2222

Table 2: Comparison of results

\begin{tabular}{|c|c|c|c|c|c|c|c|}
\hline \multicolumn{2}{|c|}{ Models } & $\begin{array}{c}\text { NB } \\
\text {-KNN }\end{array}$ & $\begin{array}{c}\text { Non- } \\
\text { linear } \\
\text { multi- } \\
\text { domain }\end{array}$ & $\begin{array}{c}\text { Deep } \\
\text { stacked }\end{array}$ & $\begin{array}{c}\text { AWG0 } \\
\text { deep } \\
\text { stacked }\end{array}$ & $\begin{array}{c}\text { WDS - } \\
\text { ENR } \\
\text { model }\end{array}$ & $\begin{array}{c}\text { BP- } \\
\text { ASE } \\
\text { and } \\
\text { ResNet }\end{array}$ \\
\hline \multirow{2}{*}{$\begin{array}{c}\text { Training } \\
\text { data }\end{array}$} & Accuracy & 87.9623 & 91.9692 & 92.6 & 94.1035 & 98.78 & 99.396 \\
\cline { 2 - 8 } & Specificity & 88.8321 & 91.243 & 91.8 & 91.9832 & 92.32 & 95.38 \\
\cline { 2 - 8 } & Sensitivity & 58.6483 & 84.41 & 97.22 & 97.3456 & 98.52 & 99.12 \\
\hline \multirow{2}{*}{$\begin{array}{c}\text { K-fold } \\
\text { data }\end{array}$} & Accuracy & 92.1374 & 93.26 & 93.45 & 93.6431 & 97.732 & 99.41 \\
\cline { 2 - 8 } & Specificity & 91.2389 & 91.56 & 91.8 & 91.984 & 95.74 & 96.12 \\
\cline { 2 - 8 } & Sensitivity & 92.6552 & 92.8 & 92.3 & 93 & 96.94 & 97.13 \\
\hline
\end{tabular}

results

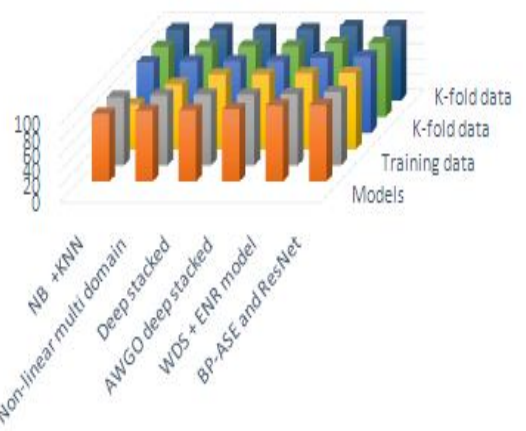

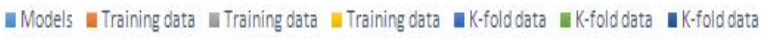

Figure 14: Comparison of results

Table 2 \& figure 14 explains about various methods, and it performs measures, at all stage of parameters the proposed BP-ASE and ResNet deep learning model achieves more improvement compared to existed methods.

Table 3: True positive rate and Error

\begin{tabular}{|c|c|c|c|c|}
\hline Parameter & $\begin{array}{c}\text { Deep } \\
\text { stacked }\end{array}$ & AGWO & DWS+ENR & $\begin{array}{c}\text { BP-ASE } \\
\text { and } \\
\text { ResNet }\end{array}$ \\
\hline $\begin{array}{c}\text { True } \\
\text { positive } \\
\text { rate }\end{array}$ & 0.872 & 0.912 & 0.943 & 0.971 \\
\hline $\begin{array}{c}\text { F1 score } \\
\text { MNSE }\end{array}$ & 0.893 & 0.934 & 0.951 & 0.968 \\
\hline & 0.04 & 0.01 & 0.001 \\
\hline
\end{tabular}



Figure 15: Performance measures

figure 15 and table 3 demonstrate that MNSE, $\mathrm{F}_{1}$ score and True positive rate analysis, in this at all stages BP-ASE and ResNet archives more improvement compared to the existed method.

\section{CONCLUSION}

In this research, implemented a BP_ASE and ResNet deep learning technique for the automatic prior stage of epileptic seizers identification. This investigation simplifies the diagnosis process by using patients EEG-dataset. It is an unsupervised learning procedure for continuous monitoring of clinical dataset. The involuntary testing-training method has used to notice the seizures in the EEG dataset. If any suspicious intervals identified in the EEG signal, which has straight fed into ResNet classifier for diagnosis. The proposed ResNet classifier experiment outperforms the existed methods; the performs measures such as accuracy is $99.4 \%$, specificity $96 \%$ and sensitivity $99 \%$ has been achieved. The other more performance outcomes True positive rate $97.1 \% \mathrm{~F} 1$ score $96.8 \%$ and MNSE is 0.001 attained. This research had conducted on public sowjanay general hospital patients data and CHB-MIT dataset. This advanced design is a robust method with classification accuracy, specificity, True positive rate and sensitivity. This technique also identifies the general, epilepsy and other abnormalities that are not included in our research. This work is challenging the external applications when diagnosis the epilepsy patients.

\section{REFERENCES}

1. X. Zhu, H. I. Suk, L. Wang, S.-W. Lee, D. Shen, and Alzheimer's Disease Neuroimaging Initiative, "A novel relational regularization feature selection method for joint regression and classication in $\mathrm{AD}$ diagnosis," Med. Image Anal., vol. 38, pp. 205-214, May 2017. https://doi.org/10.1016/j.media.2015.10.008

2. H. Niu, I. A' lvarez A' lvarez, F. Guille'n-Grima, and I. Aguinaga-Ontoso, "Prevalencia e incidencia de la enfermedad de alzheimer en europa: metaan'alisis," Neurologia, vol. 32, no. 8, p. 523532, 2017. 
3. World Alzheimer Report 2018-The State of the Art of Dementia Research: New Frontiers, Alzheimer's Disease Int., London, U.K., Sep. 2018.

4. R. J. Perrin, A. M. Fagan, and D. M. Holtzman, "Multimodal techniques for diagnosis and prognosis of Alzheimer's disease," Nature, vol. 461, pp. 916-922, Oct. 2009. https://doi.org/10.1038/nature08538

5. J. Barnesa, B. C. Dickerson, C. Frost, L. C. Jiskoot, D. Wolk, and W. M. van der Flier, “Alzheimer's disease rst symptoms are age dependent: Evidence from the NACC dataset," Alzheimer's Dementia, vol. 11, no. 11, pp. 1349-1357, Nov. 2015. https://doi.org/10.1016/j.jalz.2014.12.007

6. P. Maresova, H. Mohelska, J. Dolejs, and K. Kuca, "Socio-economic aspects of Alzheimer's disease," Curr. Alzheimer Res., vol. 12, no. 9, pp. 903-911, 2015.

7. R. A. Sperling, P. S. Aisen, L. A. Beckett, D. A. Bennett, S. Craft, A. M. Fagan, T. Iwatsubo, C. R. Jack, Jr., J. Kaye, T. J. Montine, D. C. Park, E. M. Reiman, C. C. Rowe, E. Siemers, Y. Stern, K. Yaffe, M, C. Carrillo, B. Thies, and C. H. Phelps, "Toward de-ning the preclinical stages of Alzheimer's disease: Recommendations from the National Institute on Aging-Alzheimer's Association workgroups on diagnostic guidelines for Alzheimer's disease," Alzheimer's Dementia, vol. 7, no. 3, pp. 280-292, May 2011. https://doi.org/10.1016/j.jalz.2011.03.003

8. H.-I. Suk, S. Lee, D. Shen, and Alzheimer's Disease Neuroimaging Initiative, "Hierarchical feature representation and multimodal fusion with deep learning for $\mathrm{AD} / \mathrm{MCI}$ diagnosis," NeuroImage, vol. 101, pp. 569-582, Nov. 2014.

9. H.-I. Suk and D. Shen, "Deep learning-based feature representation for AD/MCI classification," Med. Image Comput. Comput. Assist. Intervent., vol. 16, no. 2, pp. 583-590, 2013.

10. H. Khan, L. Marcuse, M. Fields, K. Swann, and B. Yener, "Focal onset seizure prediction using convolutional networks," IEEE Trans. Biomed. Eng., vol. 65, no. 9, pp. 2109-2118, Sep. 2018.

11. K. Fei, W. Wang, Q. Yang, and S. Tang, "Chaos feature study in fractional Fourier domain for preictal prediction of epileptic seizure,' Neurocomputing, vol. 249, pp. 290-298, Aug. 2017.

https://doi.org/10.1016/j.neucom.2017.04.019

12. F. Ibrahim, S. Abd-Elateif El-Gindy, S. M. El-Dolil, A. S. El-Fishawy, E.-S.-M. El-Rabaie, M. I. Dessouky, I. M. Eldokany, T. N. Alotaiby, S. A. Alshebeili, and F. E. Abd El-Samie, "A statistical framework for EEG channel selection and seizure prediction on mobile," Int. J. Speech Technol., vol. 22, no. 1, pp. 191-203, Jan. 2019.

13. T. N. Alotaiby, S. A. Alshebeili, F. M. Alotaibi, and S. R. Alrshoud, "Epileptic seizure prediction using CSP and LDA for scalp EEG signals," Comput. Intell. Neurosci., vol. 2017, pp. 1-11, Oct. 2017.

https://doi.org/10.1155/2017/1240323
14. D. Cho, B. Min, J. Kim, and B. Lee, "EEG-based prediction of epileptic seizures using phase synchronization elicited from noise-assisted multivariate empirical mode decomposition," IEEE Trans. Neural Syst. Rehabil. Eng., vol. 25, no. 8, pp. 1309-1318, Aug. 2017.

15. M. H. Myers, A. Padmanabha, G. Hossain, A. L. de Jongh Curry, and C. D. Blaha, "Seizure prediction and detection via phase and amplitude lock values," Frontiers Human Neurosci., vol. 10, p. 80, Mar. 2016. https://doi.org/10.3389/fnhum.2016.00080

16. S. Otoum, M. Ahmed, and H. T. Mouftah, "Sensor medium access control (SMAC)-based epilepsy patients monitoring system,' in Proc. IEEE 28th Can. Conf. Electr. Comput. Eng. (CCECE), May 2015, pp. 1109-1114.

17. A. de Jongh, J. C. de Munck, S. I. Gonçalves, and P. Ossenblok, "Differences in MEG/EEG epileptic spike yields explained by regional differences in signal-to-noise ratios," J. Clin. Neurophysiol., vol. 22, no. 2, pp. 153-158, Apr. 2005.

18. J. Rasekhi, M. R. K. Mollaei, M. Bandarabadi, C. A. Teixeira, and A. Dourado, "Preprocessing effects of 22 linear univariate features on the performance of seizure prediction methods," J. Neurosci. Methods, vol. 217, nos. 1-2, pp. 9-16, Jul. 2013. https://doi.org/10.1016/j.jneumeth.2013.03.019

19. M. Bandarabadi, C. A. Teixeira, J. Rasekhi, and A. Dourado, "Epileptic seizure prediction using relative spectral power features," Clin. Neurophysiol., vol. 126, no. 2, pp. 237-248, Feb. 2015.

20. K. K. Ang, Z. Y. Chin, C. Wang, C. Guan, and H. Zhang, "Filter bank common spatial pattern algorithm on BCI competition IV datasets $2 \mathrm{~A}$ and $2 \mathrm{~B}$,' Frontiers Neurosci., vol. 6, p. 39, Mar. 2012. https://doi.org/10.3389/fnins.2012.00039

21. S. M. Usman, S. Khalid, R. Akhtar, Z. Bortolotto, Z. Bashir, and H. Qiu, "Using scalp EEG and intracranial EEG signals for predicting epileptic seizures: Review of available methodologies," Seizure, vol. 71, pp. 258-269, Oct. 2019. https://doi.org/10.1016/j.seizure.2019.08.006

22. S. M. Usman, M. Usman, and S. Fong, "Epileptic seizures prediction using machine learning methods," Comput. Math. Methods Med., vol. 2017, pp. 1-10, Dec. 2017. https://doi.org/10.1155/2017/9074759

23. A. Antoniades, L. Spyrou, C. C. Took, and S. Sanei, "Deep learning for epileptic intracranial EEG data," in Proc. IEEE 26th Int. Workshop Mach. Learn. Signal Process. (MLSP), Sep. 2016, pp. 1-6. https://doi.org/10.1109/MLSP.2016.7738824 